\title{
NONLINEAR FINITE ELEMENT ANALYSIS OF NON- SEISMICALLY DETAILED INTERIOR RC BEAM-COLUMN CONNECTION UNDER REVERSED CYCLIC LOAD
}

\author{
Teeraphot Supaviriyakit*, Amorn Pimanmas \\ Sirindhorn International Institute of Technology, \\ Thammasat University, Pathumthani 12121, Thailand \\ Pennung Warnitchai \\ School of Civil Engineering, \\ Asian Institute of Technology, Pathumthani 12120, Thailand \\ Received 05 June 2006
}

\begin{abstract}
This paper presents a nonlinear finite element analysis of non-seismically detailed RC beamcolumn connections under reversed cyclic load. The test of half-scale nonductile reinforced concrete beam-column joints was conducted. The tested specimens represented those of the actual mid-rise reinforced concrete frame buildings designed according to the non-seismic provisions of the ACI building code. The test results show that specimens representing small and medium column tributary area failed in brittle joint shear while specimen representing large column tributary area failed by ductile flexure though no ductile reinforcement details were provided. The nonlinear finite element analysis was applied to simulate the behavior of the specimens. The finite element analysis employs the smeared crack approach for modeling beam, column and joint, and employs the discrete crack approach for modeling the interface between beam and joint face. The nonlinear constitutive models of reinforced concrete elements consist of coupled tension-compression model to model normal force orthogonal and parallel to the crack and shear transfer model to capture the shear sliding mechanism. The FEM shows good comparison with test results in terms of load-displacement relations, hysteretic loops, cracking process and the failure mode of the tested specimens. The finite element analysis clarifies that the joint shear failure was caused by the collapse of principal diagonal concrete strut.
\end{abstract}

Keywords: Beam-column connection, nonlinear analysis, reversed cyclic load, joint shear failure, diagonal compressive strut, reinforced concrete plate element

\section{INTRODUCTION}

Although many cities in South East Asia are considered as being located in low to moderate seismic zone due to a relatively long distance from active earth faults, they are not absolutely exempt from seismic hazard. Bangkok, for example, is founded on a soft basin of marine clay of several ten-meter depths. This soil characteristic has a potential to amplify the seismic wave up to 3 - 4 times [1]. The soft ground condition is quite similar to that of Mexico City, which was

\footnotetext{
* Corresponding author e-mail: teeraphot@gmail.com
} 
devastated by 1985 Mexico earthquake with almost 10,000 death toll. Recently, the 2004 Sumatra earthquake in Andaman Sea recorded at a magnitude of 9.3 on Richter Scale, caused violent shaking of many high rises in Bangkok though the epicenter was more than 800 kilometer far away. The quake has prompted a serious public concern on seismic safety of buildings. Many tall buildings, though not designed for seismic loads, usually have shear walls to resist wind forces, and consequently may not be vulnerable to the overall collapse under the earthquake $[2,3]$. On the contrary, many low-rise and mid-rise buildings of up to 10 stories are rigid frame without shear walls. The frame structures mainly resist lateral forces through bending of beams and columns. Most of these frame structures were designed for gravity load only according to the American Concrete building code (ACI) in Thailand and British code (BS) in Singapore and Malaysia [4].

As a result of the lack of seismic consideration in structural design, the reinforcement details of these frame buildings are usually weak against earthquake loading. As shown in Fig. 1, the three distinctive insufficient details are characterized by (a) little or no confining reinforcement in beam-column joint, (b) lap splice of longitudinal column bars immediately above floor level, (c) widely-spaced column ties and beam stirrups. Under lateral force, the joint has to carry a large horizontal shear force (Fig. 2) in order to equilibrate moments acting on the joint in the same direction by framing beams. Concurrently, the longitudinal beam bar in the joint is also subject to a large bond stress. As a result of these forces, the joint commonly fails by either joint shear or bar pull-out failure.

Recently, the authors have conducted a survey of existing RC buildings in Thailand [5]. The building database was compiled for mid-rise reinforced concrete buildings having the number of stories in the region of 5 to 15 . All buildings were beam-column rigid frame without shear wall. They were designed according to the non-seismic provisions of ACI building code considering only gravity load. The buildings were grouped into 3 categories based on their column tributary area. The beam-column connections in each category were studied in terms of structural designs and reinforcement details. Three half-scale beam-column specimens that represented each building category were prepared and tested in the laboratory under reversed cyclic load. Since buildings were designed in accordance with the ACI building code, the experimental findings are supposedly applicable to not only buildings in Thailand, but also buildings designed and detailed according to the ACI code in other countries.

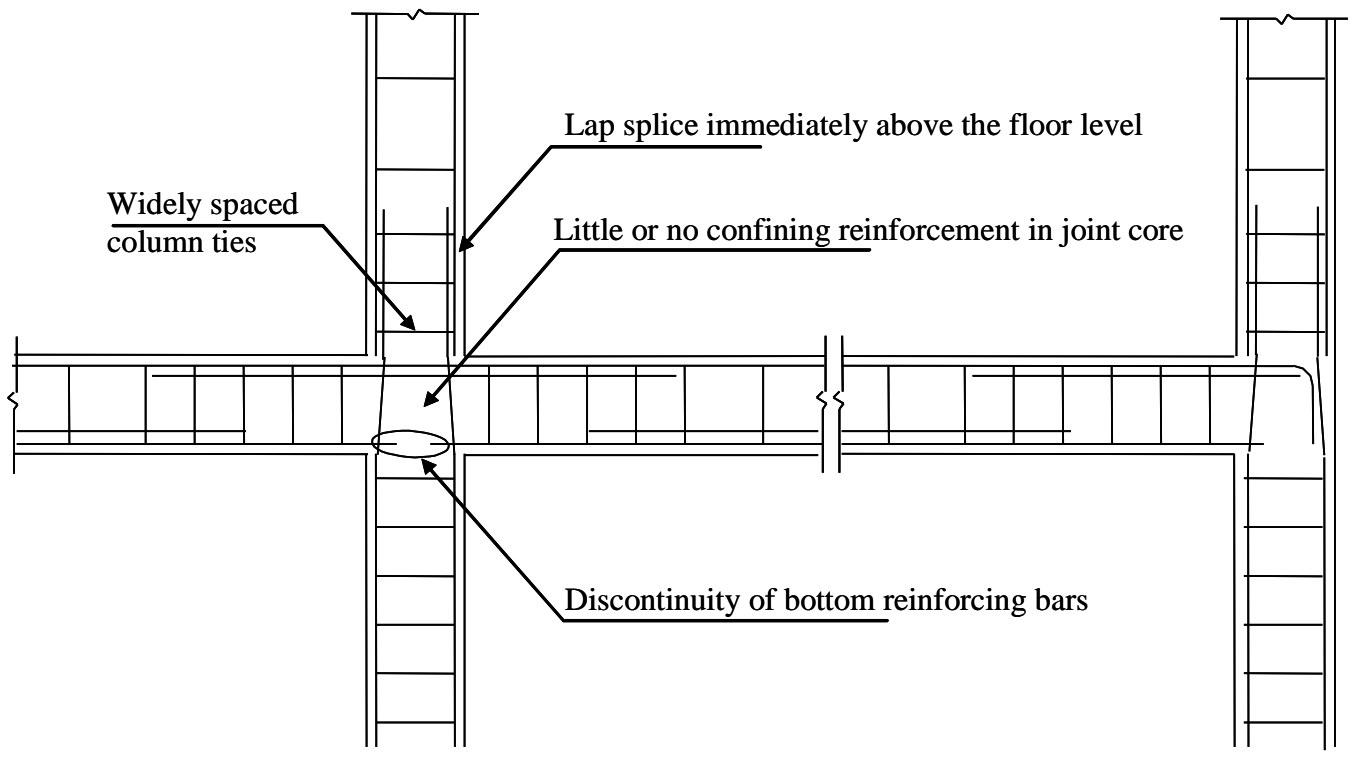

Fig. 1: Typical non-seismic reinforcing detail of $R C$ frame designed for gravity load only. 


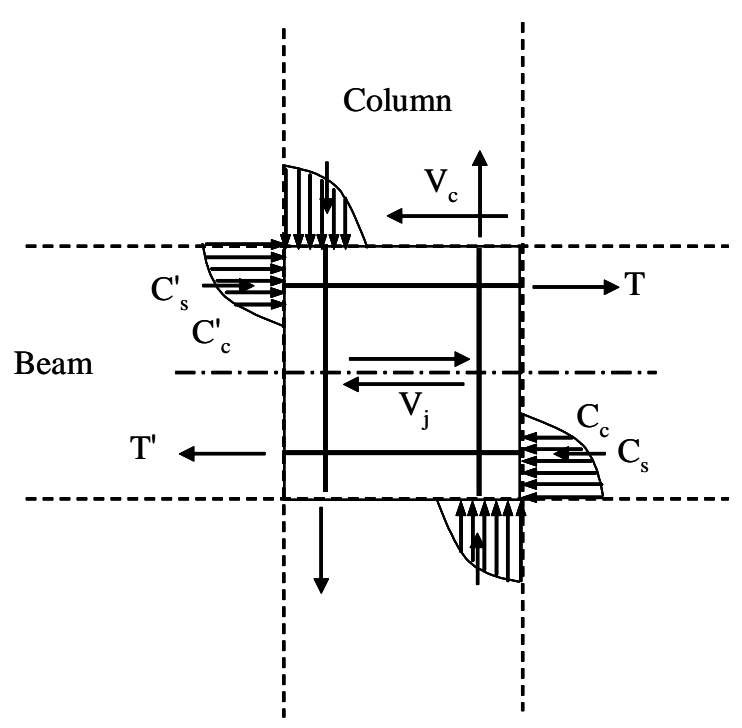

Fig. 2: Horizontal shear force in joint core.

The experiment showed a sudden shear failure in the joint region of specimen representing connection of buildings with small and medium column tributary area $\left(9-30 \mathrm{~m}^{2}\right)$ and ductile flexural failure of beam sections near the joint in the specimen representing connection of building with large column tributary area $\left(40-48 \mathrm{~m}^{2}\right)$. In this paper, the authors presented the nonlinear finite element method as a tool to simulate reversed cyclic behaviors of these specimens. The beam-column joint specimens are modeled with two-dimensional plane stress elements in line with the smeared crack approach. The interface between beam and column is modeled with one-dimensional discrete joint element to simulate reinforcement pull-out. The finite element method is used to investigate the cracking process, the failure mode, the global load-displacement behavior and the principal load resistant mechanism.

\section{NONLINEAR FINITE ELEMENT ANALYSIS OF REINFORCED CONCRETE (RC) INTERIOR BEAM-COLUMN CONNECTION}

\subsection{Finite element model}

Figure 3 illustrates a finite element model of a typical reinforced concrete (RC) interior beamcolumn connection of a rigid frame. The cruciform-shaped specimen is normally preferred for laboratory testing. It consists of the beam-column joint core with beams and columns extending horizontally and vertically to their mid-length where moments are assumed to be zero, i.e., point of inflection. The beam, column and beam-column regions are modeled with two-dimensional 8-node plane stress RC elements as shown in Fig. 4(a). The interfaces between beam and column are modeled with one-dimensional 6-node joint element as shown in Fig. 4(b).

The two-dimensional reinforced concrete element is constructed by combining the constitutive laws of concrete and reinforcing bar. Here, cracks and reinforcing bars are assumed to be distributed or smeared over the entire element. The bond between concrete and reinforcement is considered to be perfect, that is, concrete and reinforcement are subject to identical strains throughout the entire loading. The total stress carried by the reinforced concrete element is the sum of concrete and reinforcement stresses. In the smeared crack approach, the constitutive models of reinforcement and concrete are formulated in terms of relations between average stress and average strain as follows, 


$$
\begin{gathered}
\left\{\sigma_{\mathrm{T}, \mathrm{rc}}\right\}=\left\{\sigma_{\mathrm{c}, \mathrm{rc}}\right\}+\left\{\sigma_{\mathrm{r}, \mathrm{rc}}\right\} \\
\left\{\sigma_{\mathrm{T}, \mathrm{rc}}\right\}=\left\{\sigma_{\mathrm{x}}, \sigma_{\mathrm{y}}, \tau_{\mathrm{xy}}\right\}=\mathrm{f}_{\mathrm{rc}}\left(\left\{\varepsilon_{\mathrm{x}}, \varepsilon_{\mathrm{y}}, \gamma_{\mathrm{xy}}\right\}\right)+\mathrm{g}_{\mathrm{rc}}\left(\left\{\varepsilon_{\mathrm{x}}, \varepsilon_{\mathrm{y}}, \gamma_{\mathrm{xy}}\right\}\right)
\end{gathered}
$$

Where $\left\{\sigma_{\mathrm{T}, \mathrm{rc}}\right\},\left\{\sigma_{\mathrm{c}, \mathrm{rc}}\right\}$ and $\left\{\sigma_{\mathrm{r}, \mathrm{rc}}\right\}$ are total stresses, concrete stresses and reinforcement stresses of RC plate element, respectively, $\left\{\sigma_{\mathrm{x}}, \sigma_{\mathrm{y}}, \tau_{\mathrm{xy}}\right\}$ and $\left\{\varepsilon_{\mathrm{x}}, \varepsilon_{\mathrm{y}}, \gamma_{\mathrm{xy}}\right\}$ are global stress and strain, respectively, $\mathrm{f}_{\mathrm{rc}}$ and $\mathrm{g}_{\mathrm{rc}}$ represent constitutive laws of concrete and reinforcement, respectively.

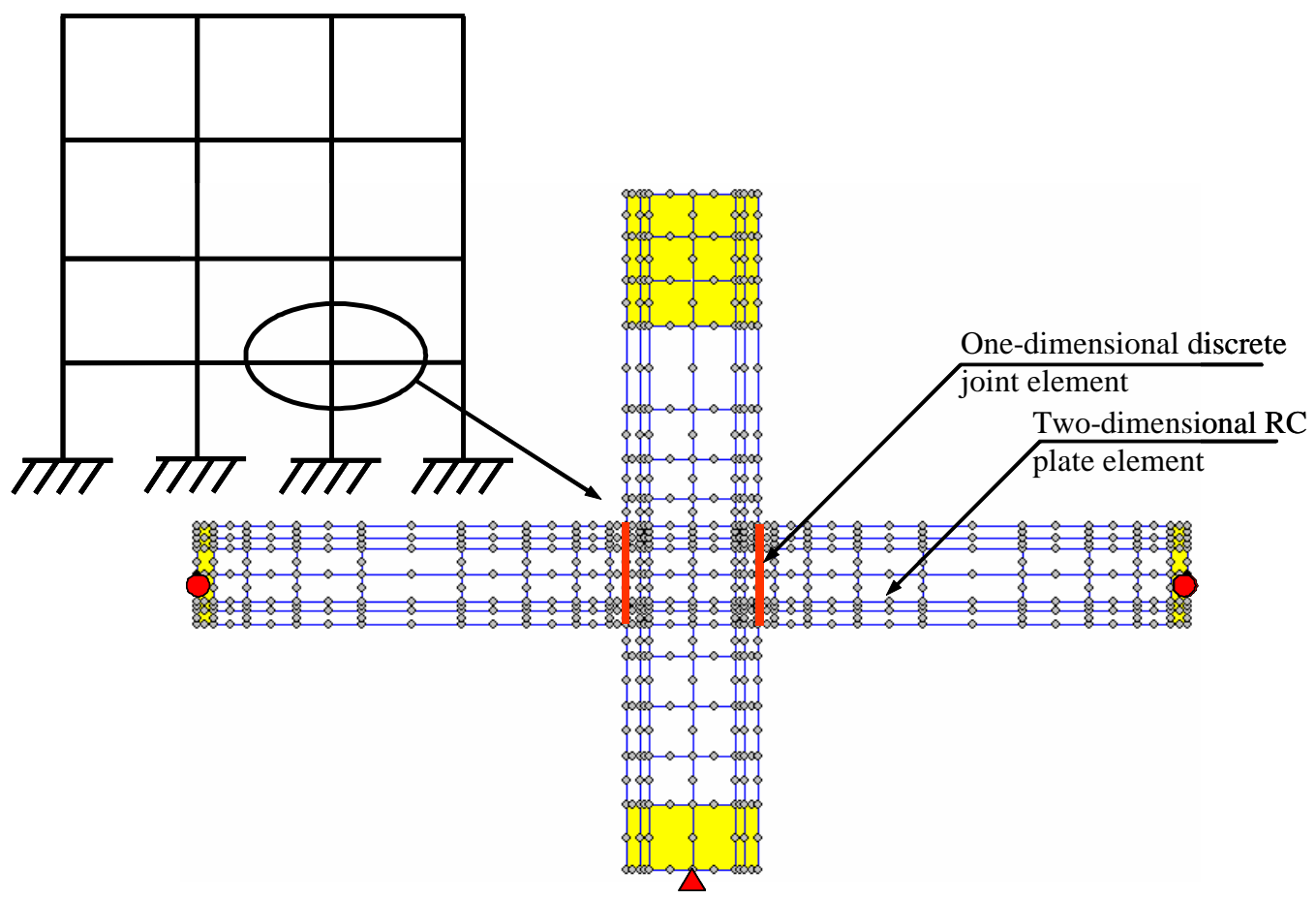

Fig. 3: Typical finite element model of RC interior beam-column connection.

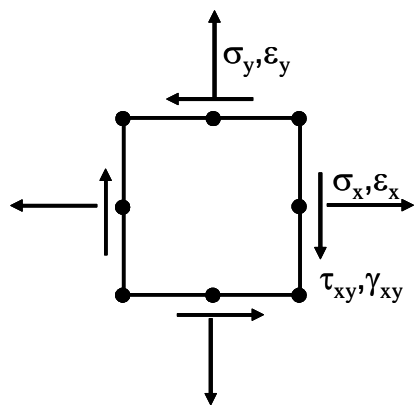

(a) Two-dimensional RC plate element

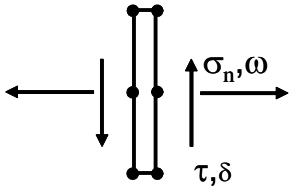

(b) One-dimensional discrete joint element

Fig. 4: Two-dimensional plate element and one dimensional joint element.

The joint element is one-dimensional with zero thickness. It represents the interface between two elements with different sectional stiffness where local discontinuities, such as reinforcement 
pull-out may occur. Similar to two-dimensional RC plate element, the total stresses of the joint element are the sum of concrete and reinforcement stresses. However, the constitutive laws of the joint element are formulated in terms of average stresses and relative displacements, i.e., crack opening and sliding.

$$
\begin{gathered}
\left\{\sigma_{\mathrm{T}, \mathrm{jo}}\right\}=\left\{\sigma_{\mathrm{c}, \mathrm{jo}}\right\}+\left\{\sigma_{\mathrm{r}, \mathrm{jo}}\right\} \\
\left\{\sigma_{\mathrm{T}, \mathrm{jo}}\right\}=\left\{\sigma_{\mathrm{n}}, \tau\right\}=\mathrm{f}_{\mathrm{jo}}(\{\omega, \delta\})+\mathrm{g}_{\mathrm{jo}}(\{\omega, \delta\})
\end{gathered}
$$

Where $\left\{\sigma_{\mathrm{T}, \text { jo }}\right\},\left\{\sigma_{\mathrm{c}, \mathrm{jo}}\right\}$ and $\left\{\sigma_{\mathrm{r}, \mathrm{jo}}\right\}$ are total stresses, concrete and reinforcement stresses of RC joint element, respectively. $\left\{\sigma_{\mathrm{n}}, \tau\right\}$ is the vector of joint normal and shear stress, $\{\omega, \delta\}$ is the vector of joint opening and sliding and, $f_{\text {jo }}$ and $g_{\text {jo }}$ represent constitutive laws of concrete and reinforcement of joint element, respectively.

Essentially, the constitutive laws for plate and joint element describe the same physical behaviors of concrete and reinforcement. However, the kinematic variables used in the constitutive formulations are different as previously explained.

The constitutive laws of concrete consist of the compressive stress model under crack closure, the tensile stress model upon crack opening and the shear stress model upon crack sliding (Fig. 5). The constitutive law of reinforcement includes the elastic-hardening model under tensile yielding and elasto-plastic model under compressive yielding. These laws represent major nonlinearity of reinforced concrete. The combined nonlinearity of all elements subject to different stress states constitutes the entire nonlinear behavior of the specimen. In the next section, only the average stress-average strain constitutive laws of the RC plate element are presented.

\subsection{Constitutive models of concrete and reinforcing bars}

\subsubsection{Cracked concrete}

The local strains of cracked concrete are defined with respect to the crack axis. They consist of strain normal to a crack, strain parallel to a crack and the shear strain along a crack face as shown in Fig. 5. Input variables for constitutive laws are local strains defined above as well as relevant path-dependent parameters. The constitutive laws are formulated for both envelope and hysteretic loops. Under cyclic load, two sets of diagonal cracks are generated and alternate opening and closing in accordance with the load direction. At any load step, however, only a set of crack is active and principally contributes the nonlinearity of the element. The active crack is defined as the one that has the largest normal tensile strain. The constitutive laws are expressed with respect to the active crack. In the following, the three previously mentioned constitutive laws are described.

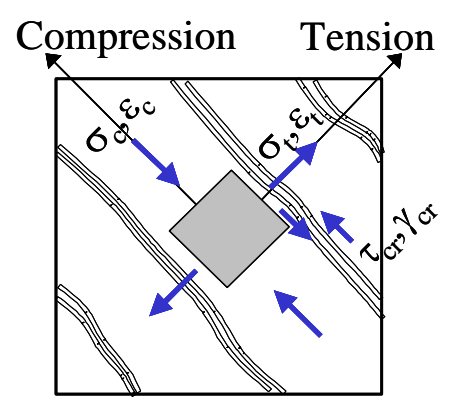

Fig. 5: Local stresses and strains of cracked concrete. 
(a) Combined compression-tension model for normal stress orthogonal and parallel to a crack

The constitutive law for computing normal stress orthogonal and parallel to a crack is shown in Fig. 6. On the tensile side, the model covers both tension softening and tension stiffening behavior. After crack occurs, the tensile stress sharply drops in the un-reinforced element while it gradually decreases in the reinforced element as a result of bond between concrete and steel bar. To represent both behaviors under the same formulation, the following equation is adopted for concrete tensile stress.

$$
\sigma_{\mathrm{t}}=\mathrm{f}_{\mathrm{t}}\left(\frac{\varepsilon_{\mathrm{tu}}}{\varepsilon_{\mathrm{t}}}\right)^{\mathrm{c}}
$$

where $\sigma_{t}, \varepsilon_{t}$ is the tensile stress and strain orthogonal to a crack, $f_{t}$ is the concrete tensile strength, $\varepsilon_{\mathrm{tu}}$ is the cracking strain, $\mathrm{c}$ is a softening/stiffening parameter. By varying the parameter $\mathrm{c}$, the above model can cover both softening and stiffening behavior. In this paper, the parameter $\mathrm{c}$ is set to 2.0 and 0.4 for un-reinforced and reinforced elements to represent softening and stiffening behavior respectively (Fig. 7).

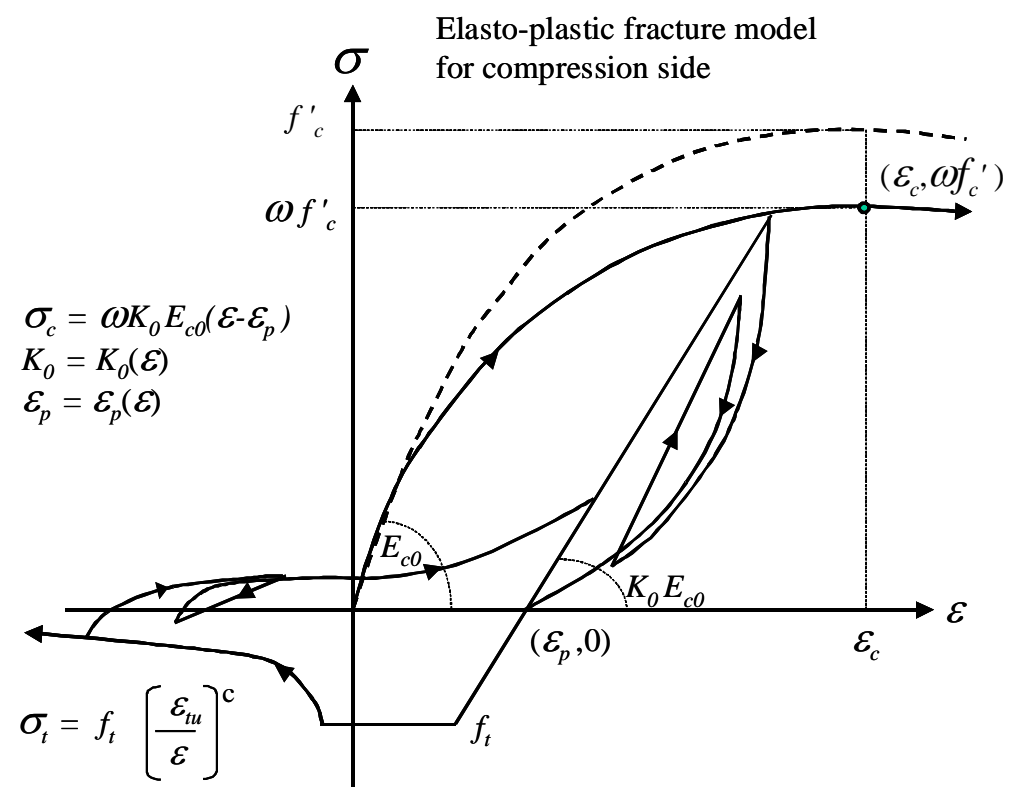

Tension stiffening / softening model for tensile side

Fig. 6: Combined compression-tension model for normal stress parallel and orthogonal to a crack.

To compute the compressive stress parallel to a crack, the following elasto-plastic fracture model [6] is used

$$
\sigma_{\mathrm{c}}=\omega \mathrm{K}_{0} \mathrm{E}_{\mathrm{c} 0}\left(\varepsilon_{\mathrm{c}}-\varepsilon_{\mathrm{p}}\right)
$$

where $\sigma_{c}, \varepsilon_{c}$ is the compressive stress and strain parallel to a crack, $\omega$ is transverse tensile strain factor, $\mathrm{K}_{0}$ is the fracture parameter, $\mathrm{E}_{\mathrm{c} 0}$ is the initial stiffness and $\varepsilon_{\mathrm{p}}$ is the compressive plastic strain. The fracture parameter $\left(\mathrm{K}_{0}\right)$ and compressive strain $\left(\varepsilon_{\mathrm{p}}\right)$ are the key parameters of the model. They are empirically formulated as [6]. 


$$
\begin{aligned}
& \mathrm{K}_{0}=\exp \left(-0.73 \frac{\varepsilon_{\mathrm{c}}}{\varepsilon^{\prime}}\left(1-\exp \left(-1.25 \frac{\varepsilon_{\mathrm{c}}}{\varepsilon^{\prime}}\right)\right)\right) \\
& \varepsilon_{\mathrm{p}}=2 \varepsilon^{\prime}\left(\frac{\varepsilon_{\mathrm{c}}}{\varepsilon^{\prime}}-\frac{20}{7}\left(1-\exp \left(-0.35 \frac{\varepsilon_{\mathrm{c}}}{\varepsilon^{\prime}}\right)\right)\right)
\end{aligned}
$$

where $\varepsilon^{\prime}$ is the concrete strain at peak compressive strength.

The model combines the nonlinearity caused by plasticity through plastic strain and fracturing damage through fracture parameter. These parameters account for the permanent deformation and irrecoverable loss of elastic strain energy, respectively. In addition, the concrete compressive strength is reduced owing to the presence of transverse tensile strain [6 - 8]. This is taken into account by an additional damage factor $(\omega)$ in equation 6 . The relation between $\omega$ and transverse tensile strain is shown in Fig. 8.

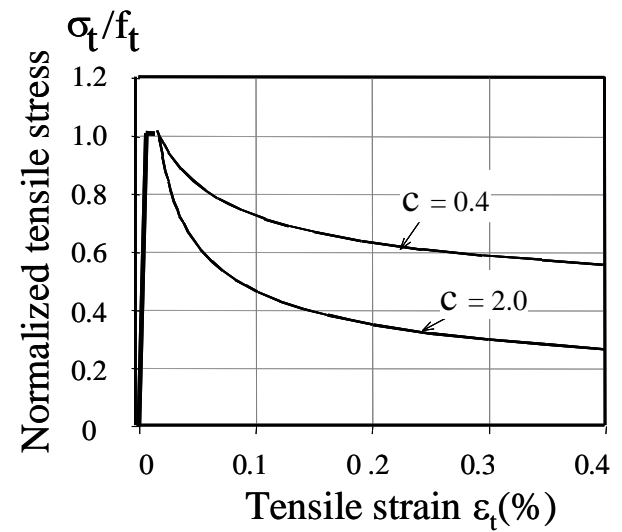

Fig. 7: Tension softening and stiffening model.

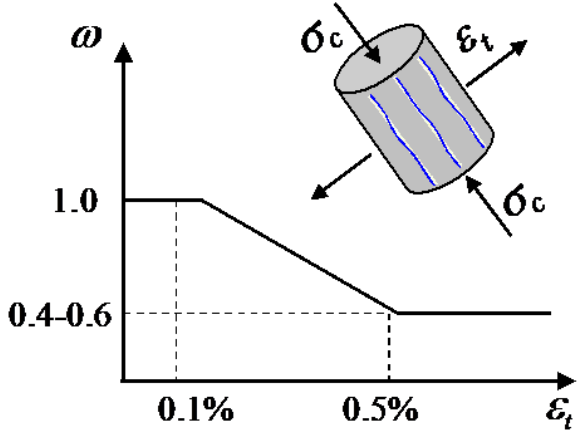

Fig. 8: Relation between $\omega$ and transverse tensile strain $\varepsilon_{t}$ ).

\section{(b) Shear stress transfer model}

For computing shear stress transmitted along a crack face, the contact density model [6] is adopted as shown in Fig. 9. The equation of the shear envelope may be expressed as,

$$
\tau_{\mathrm{cr}}=\mathrm{f}_{\mathrm{st}} \frac{\beta^{2}}{1+\beta^{2}}
$$

where $\mathrm{f}_{\mathrm{st}}$ is interface shear strength along the crack and may be expressed as,

$$
\mathrm{f}_{\mathrm{st}}=3.8\left(\mathrm{f}_{\mathrm{c}}^{\prime}\right)^{1 / 3}, \mathrm{Mpa}
$$

$\beta$ is the normalized shear strain defined as,

$$
\beta=\frac{\gamma_{\mathrm{cr}}}{\varepsilon_{\mathrm{t}}}
$$

\subsubsection{Model of reinforcing bar}

Local strains of reinforcing bar are defined with respect to the bar axis. For reinforcing steel, local yielding at the vicinity of cracks has to be taken into account in the constitutive 
formulation. Since reinforcement sections close to cracks are subject to yielding first whereas others are still elastic, that is, all reinforcement sections are not simultaneously yielding throughout the entire length. This causes the apparent reduction in the average yield strength of reinforcing bars. The average yield strength of reinforcing bars embedded in concrete may be expressed as [9],

$$
\overline{\mathrm{f}}_{\mathrm{y}}=\mathrm{f}_{\mathrm{y}}-\frac{\mathrm{f}_{\mathrm{t}}}{2 \rho}
$$

Where $\overline{\mathrm{f}}_{\mathrm{y}}=$ average yield strength of reinforcing bar embedded in concrete, $\mathrm{f}_{\mathrm{y}}$ is the yield strength of bare bar, $f_{t}$ is the tensile strength of concrete and $\rho$ is the reinforcement ratio.

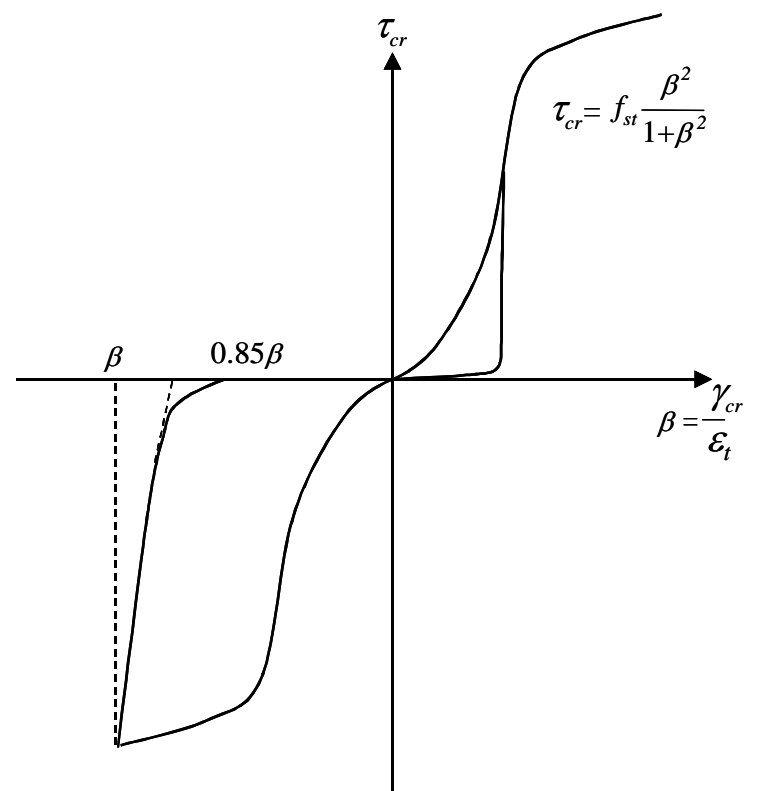

Fig. 9: Shear stress transfer model.

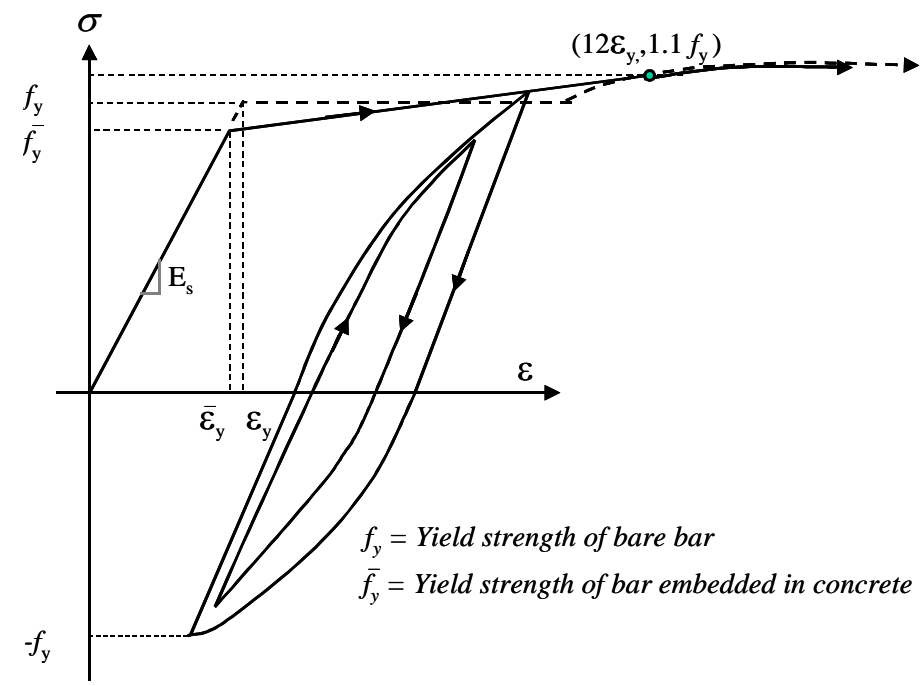

Fig. 10: Modeling of reinforcing bar. 
The model of reinforcing bar for both envelope and hysteretic loops [6] is shown in Fig. 10. The figure also shows that of bare bar for comparison.

\section{NUMERICAL SIMULATION OF RC BEAM-COLUMN CONNECTION}

\subsection{Test of non-seismically detailed interior beam-column connection}

The authors conducted the reversed cyclic test of three half scale interior beam-column joint specimens. These specimens represented those of actual buildings designed and detailed according to the non-seismic provisions of ACI building code. The database of ten reinforced concrete mid-rise buildings in Bangkok was compiled. These buildings have 5 - 15 stories and were constructed as reinforced concrete frame without shear wall. The types of buildings cover university, school, apartment and hospital. The buildings were grouped into three categories based on column tributary area (Fig. 11), as buildings with large, medium and small column tributary area. Based on the collected data, the area range is set to $40-48 \mathrm{~m}^{2}, 20-30 \mathrm{~m}^{2}$ and 9 $18 \mathrm{~m}^{2}$ for large, medium and small category, respectively. In order to characterize the structural behavior of beam-column connection, the structural indices were defined for beam, column and beam-column joint. The structural indices of beam included tension and compression reinforcement ratio, shear span-to-depth ratio, flexural capacity-to-shear capacity ratio, transverse reinforcement ratio. The structural indices of column included axial force ratio, shear span-to-depth ratio, flexural capacity-to-shear capacity ratio, longitudinal reinforcement ratio, transverse reinforcement ratio. The structural indices of beam-column joint included column depth-to-bar diameter ratio, column width-to-beam width ratio, column depth-to-beam depth ratio, confinement reinforcement index, column flexural capacity-to-beam flexural capacity ratio, joint shear-to-joint shear strength ratio.

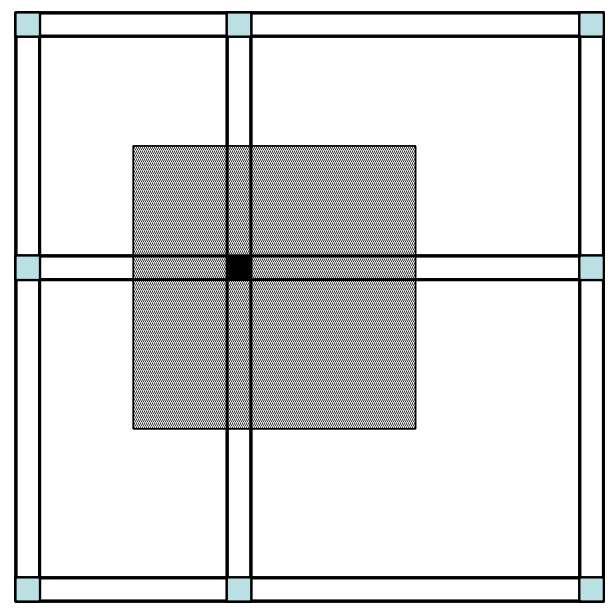

\begin{tabular}{cc}
\hline Area $\left(\mathrm{m}^{2}\right)$ & Category \\
\hline $9-18$ & Small tributary area \\
\hline $20-30$ & Medium tributary area \\
\hline $40-48$ & Large tributary area \\
\hline
\end{tabular}

Fig. 11: Definition of column tributary area.

These structural indices were calculated for each building in each category. Three half-scale beam-column specimens, namely, JL, JM and JS were constructed to represent those of each building category. The structural indices of these specimens were designed to be as close as possible to the mean values calculated from the actual building in each category. The reinforcement details and the construction methods were also as similar as possible to the actual construction. The specimen dimensions and reinforcement details are illustrated in Figs. 12 - 13. The $12 \mathrm{~mm}$ diameter reinforcing bar was used as longitudinal reinforcements in beam and 
column. The average tested yield and tensile strengths of the bar were 499 and $615 \mathrm{MPa}$, respectively. The $3 \mathrm{~mm}$ diameter plain mild steel was used as transverse reinforcements in beam and column. The tested yield and tensile strengths of the bar were 291 and $339 \mathrm{MPa}$, respectively. The average tested cylindrical compressive strength of concrete was $26.7 \mathrm{MPa}$.

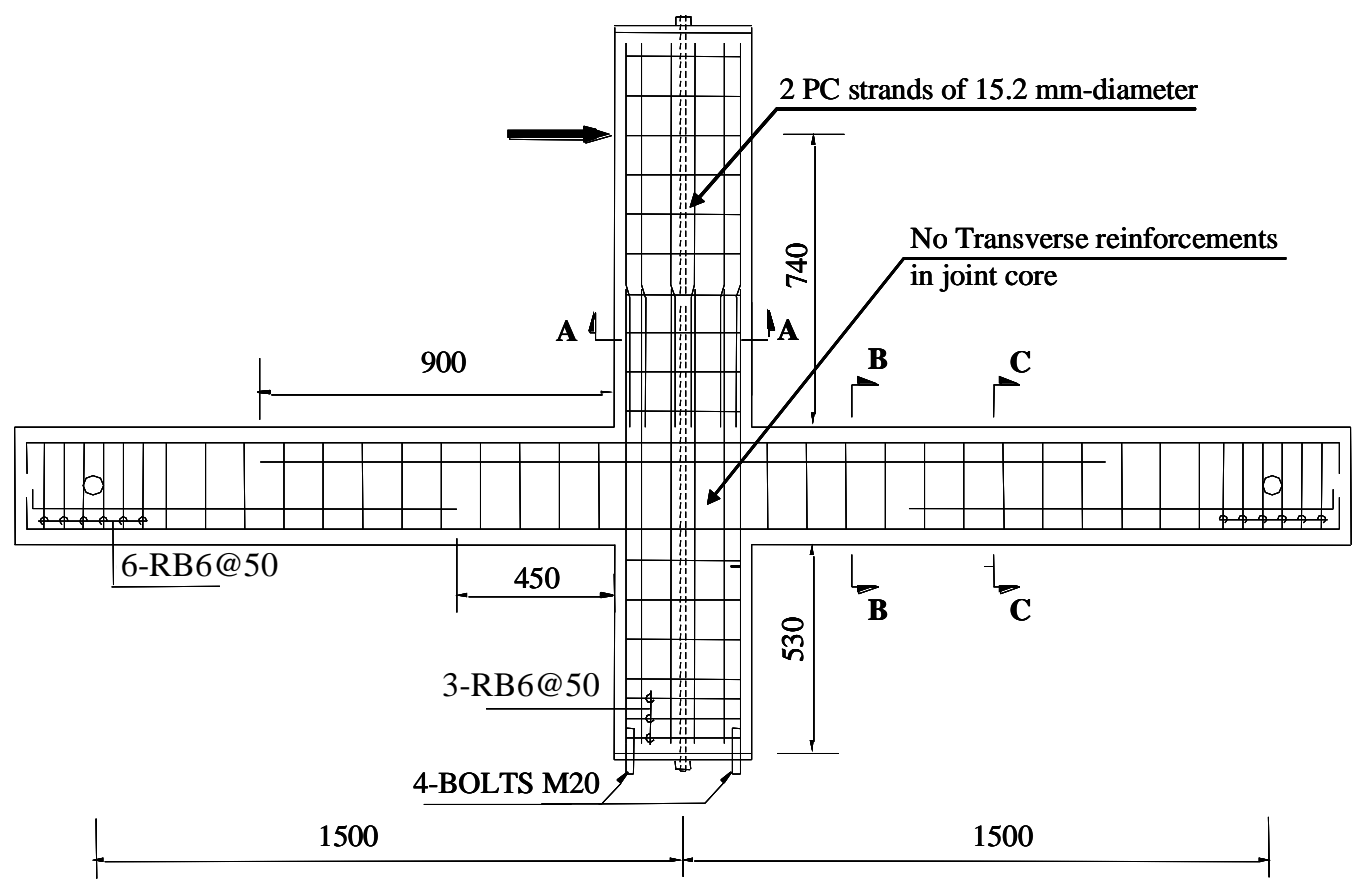

Fig. 12: Geometry, dimension and reinforcement of all specimens (unit: $\mathrm{mm}$ ).

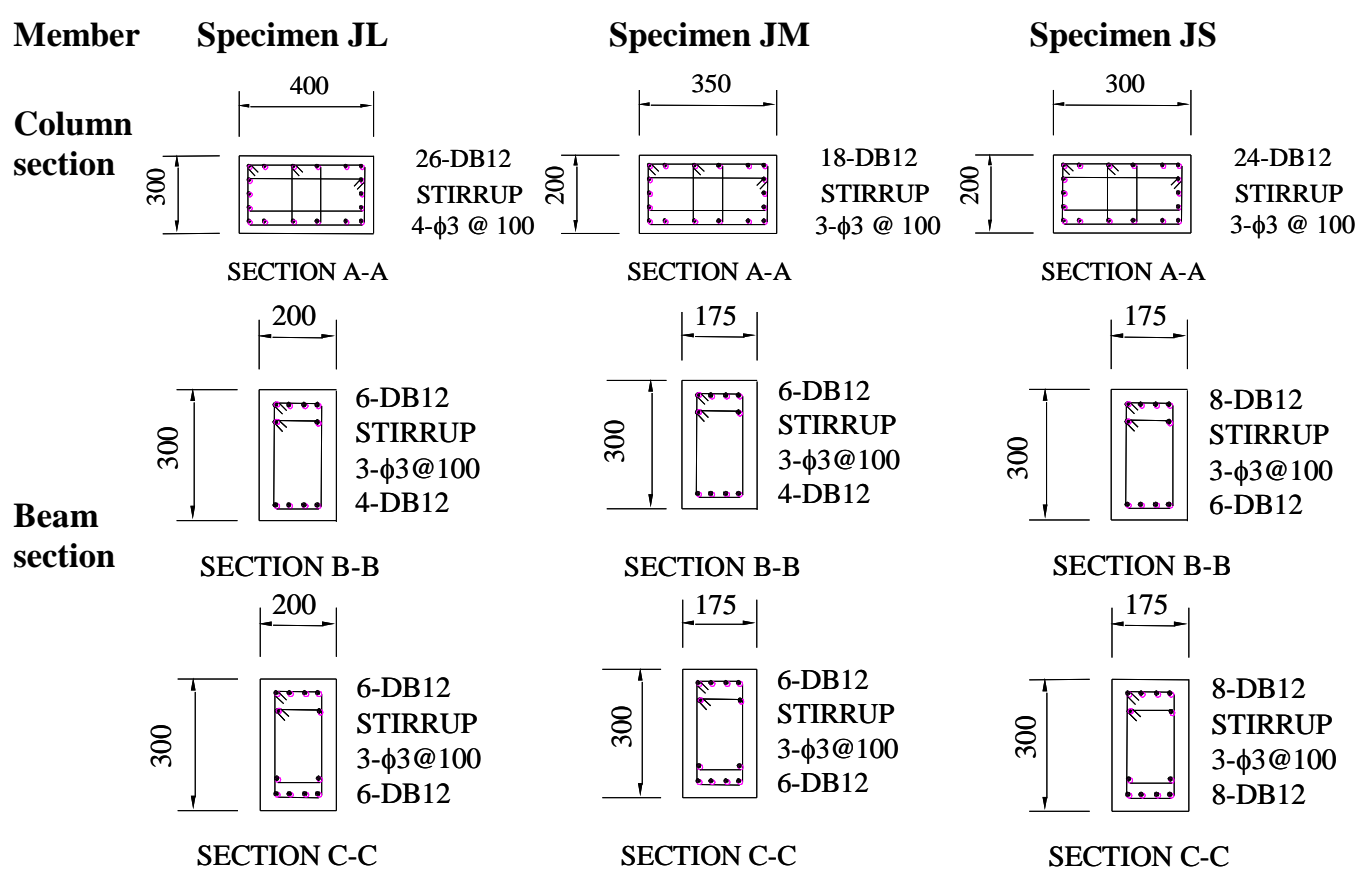

Fig. 13: Cross section of beams and columns (unit: $\mathrm{mm}$ ) . 
The test set-up and boundary conditions are shown in Fig. 14. The lateral forced displacement was applied at the top of the column through a $500 \mathrm{kN}$ hydraulic actuator. The ends of beam were supported by rollers that allowed free horizontal movement to simulate lateral drift. The bottom end of the column was supported by a hinge which allowed no movement in all directions. The axial load of $12.5 \%$ of column axial capacity was applied to the column by means of vertical prestressing. The column was pushed forward and pulled backward in a reversed cyclic pattern with the target lateral drifts of $0.25,0.50$, and $0.75 \% \ldots$ as shown in Fig. 15. The target loop was repeated twice for each drift level. The load was continued until and beyond the peak load to trace the post-peak behavior. The experimental results shall be presented and discussed in conjunction with the numerical analysis in the next section.

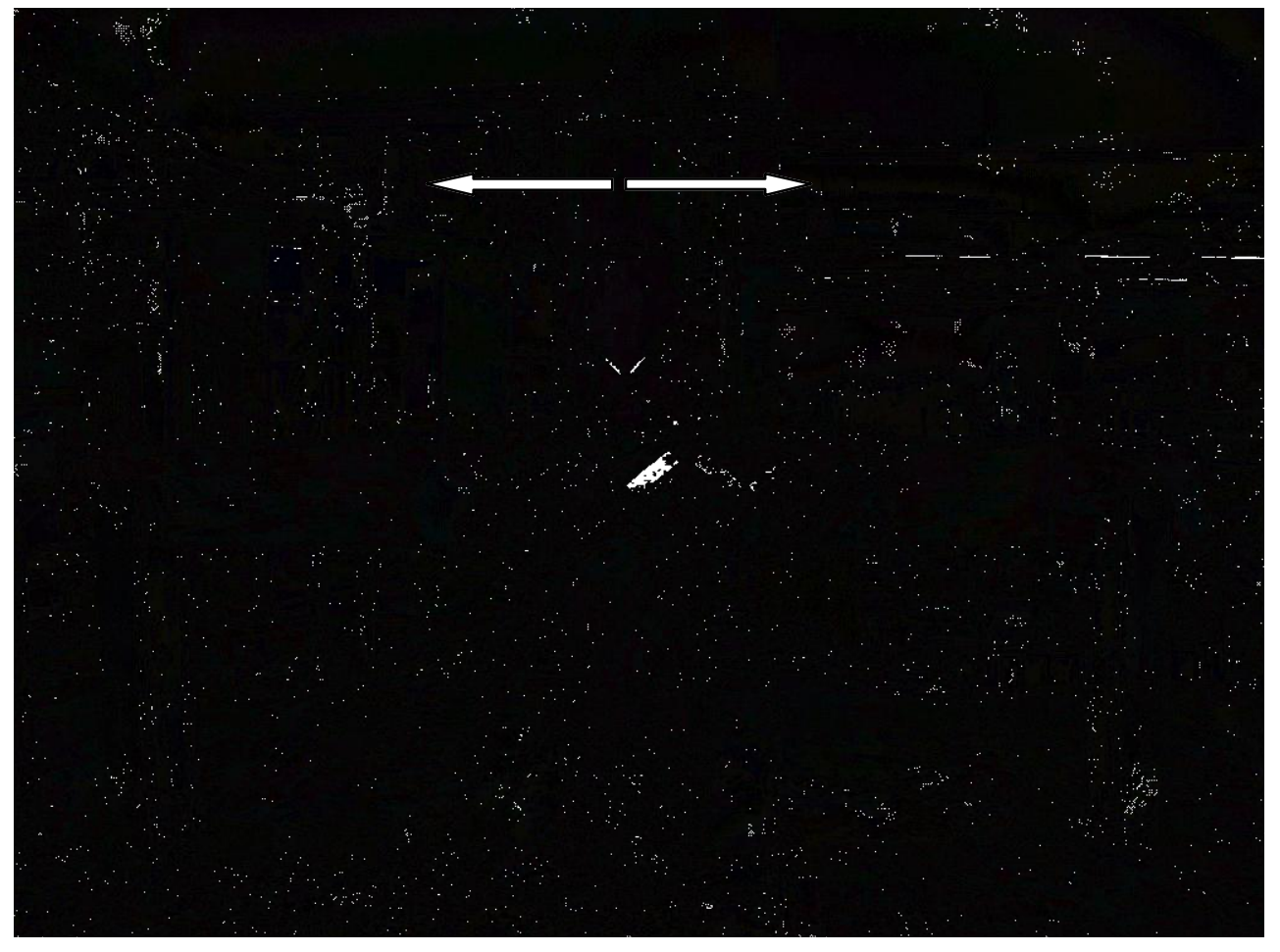

Fig. 14: Laboratory test set-up.

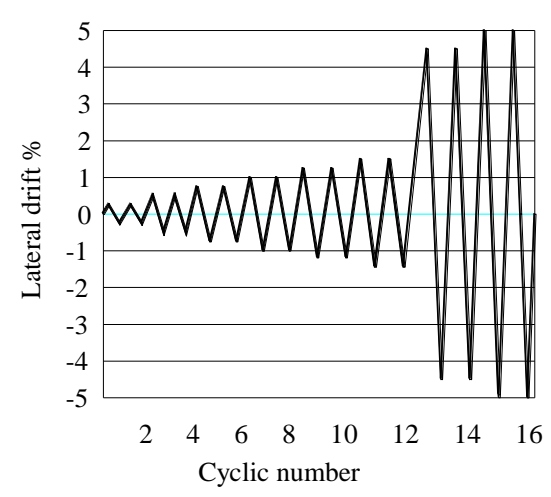

(a) Specimen JL

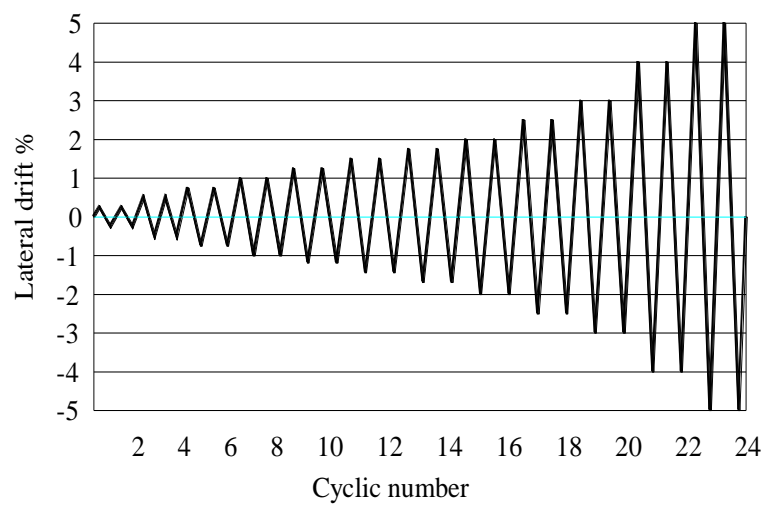

(b) Specimen JM and JS

Fig. 15: Displacement history of each specimen. 


\subsection{Finite element analysis}

The typical finite element mesh of the specimen is shown in Fig. 3. For each specimen, the mesh is composed of 733 nodes, 216 two-dimensional RC plate elements and 12 onedimensional joint elements. Loads acting on specimens include self-weight, column axial load and horizontal forced displacement. The comparison between experiment and analysis is given Table 1. The failure is defined as the point where the applied load drops more than $80 \%$.

The analytical deformed shape and cracking pattern of specimen JL are compared with the experimental results in Fig. 16. The FEM demonstrates extensive deformation at the ends of beam framing into the joints, indicating flexural failure. The evident opening of the discrete joint element indicates pull-out of beam reinforcing bars. The FEM also shows concentration of vertical cracks near the ends of beams. This crack pattern indicates that bending moments act on the beam ends in the same direction. On the contrary, the FEM predicts little distortion and cracking in the elements of the joint region. Hence failure does not originate from the joint. Moreover, no major cracks are computed in the column adjacent to the joint core. The failure is therefore categorized as ductile strong column-weak beam. In a building with large column tributary area, the size of column is usually large whereas the span of beam is long. This configuration leads to large column flexural capacity, high joint shear strength and low beam flexural strength.

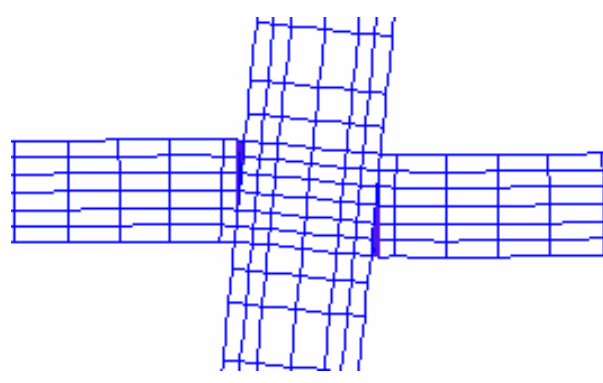

(a) Analytical deformation

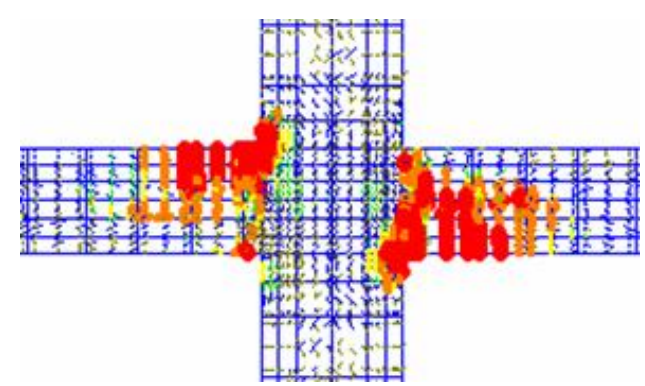

(b) Analytical cracking pattern

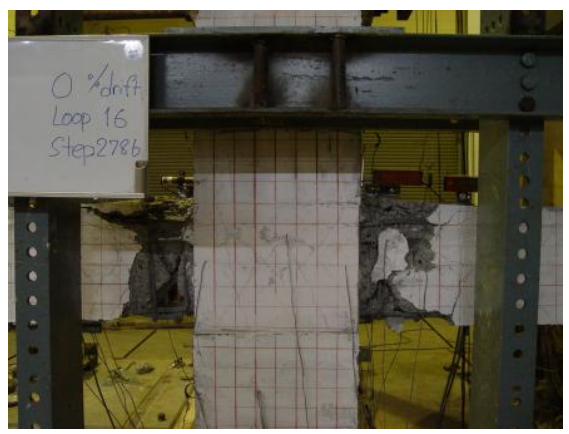

(c) Experiment

Fig. 16: Specimen JL at the end of test.

The comparison between analytical and experiment load versus applied displacement is shown in Fig. 17. A reasonable match is obtained for both envelope and hysteretic loops. The hysteretic loops are wide, indicating large energy dissipation. In the experiment, concrete crushing and subsequent spalling occurred in the beam and consequently exposed longitudinal and transverse reinforcements. This was followed by buckling of compression reinforcement. 
The spalling and reinforcement buckling are not included in the present constitutive models, hence the numerical loops are relatively wider than the experimental ones. The FEM predicts ductile yielding with long yield plateau up to $4.6 \%$ drift which is the same as the experimental value. It shall be noticed that the sub-standard beam-column joint in buildings with large column tributary area is quite ductile though it was designed and detailed without considering seismic loads in accordance with the ACI building code. The ductility can be enhanced by providing closed stirrups near the beam's end to prevent concrete spalling and reinforcement buckling.

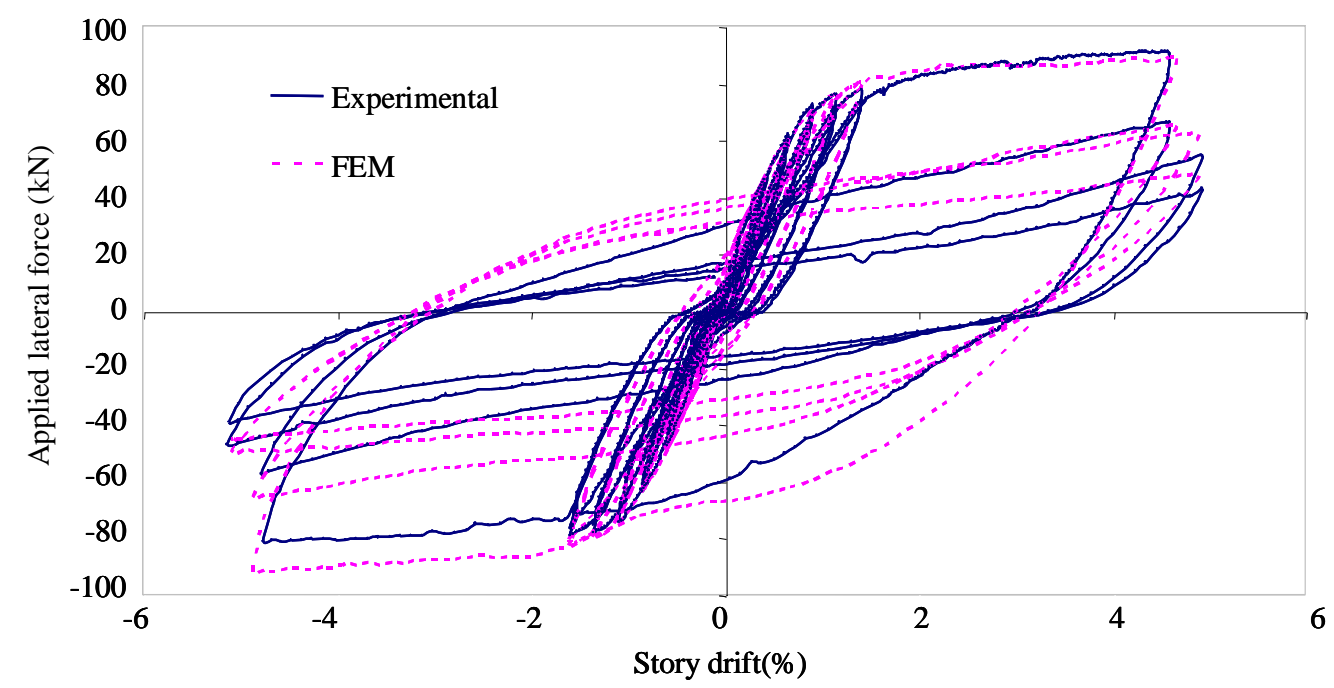

Fig. 17: Relationship between applied load and displacement of Specimen JL.

The analytical deformed shape and cracking pattern of specimen JM and JS are compared with experimental results in Figs. 18 and 20, respectively. In contrast to specimen JL, the mesh of JM and JS is extremely distorted, showing large shear deformation in the joint region. The analytical cracks are mostly inclined and concentrated in the joint zone. Two sets of intersecting diagonal cracks alternate opening and closing in accordance with the change in the load direction. These cracks form the dominant X-shape pattern. The analytical joint damage and development of diagonal cracks closely follow experimental results. Some small flexural cracks are computed in the beams and columns but do not cause the failure. The failure originates from the joint zone as a result of excessive joint shear force.

The comparison between experimental and numerical load versus applied displacement relation is shown in Figs. 19 and 21 for specimen JM and JS, respectively. The maximum experimental drifts at peak load are 1.75 and $1.5 \%$ for specimens JM and JS, respectively. The corresponding computed drifts are 2.0 and $1.5 \%$, respectively. The hysteretic loops are narrow and pinched towards the origin, indicating small energy dissipation. This may be associated with sliding of diagonal cracks in the joint. However, the analytical loops are wider than experimental ones. This is supposedly caused by the concrete spalling in the joint area which is not included in the present analytical model. It is also observed that the attained lateral drift tends to be smaller for substandard beam-column joint in RC frame with smaller tributary column. This is probably because the frame is likely to have smaller column dimensions, just sufficient to carry gravity load.

The joint shear failure is undesirable because it is brittle and catastrophic. The tendency of brittleness is more evident for beam-column connection with smaller column tributary area. To analytically identify the failure mechanism of the joint region, the principal compressive stresses 
of concrete are computed from the FEM stress outputs. The development of compressive struts is compared among the three analyzed specimens in Fig. 22.

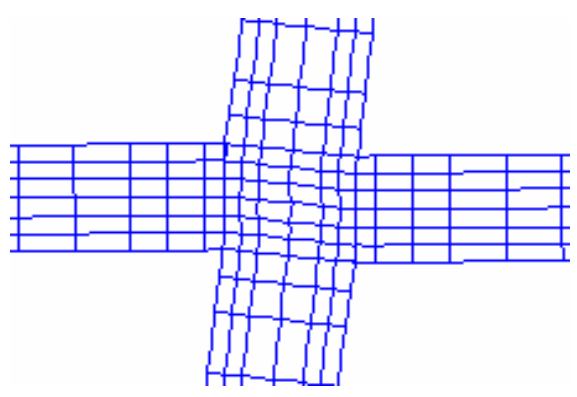

(a) Analytical deformation

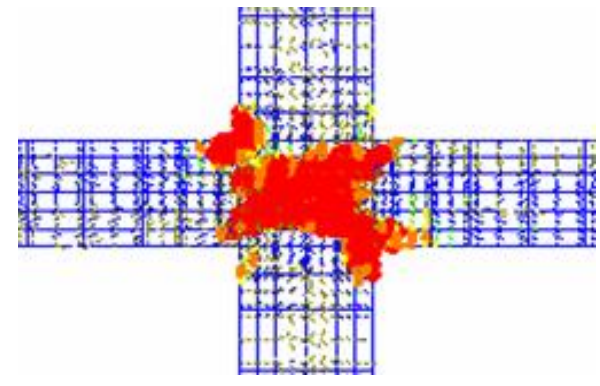

(b) Analytical cracking pattern

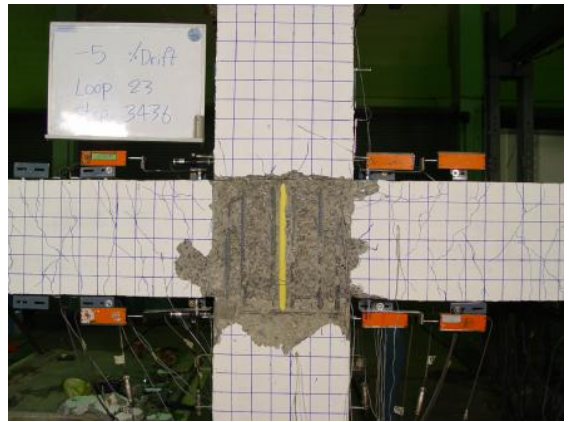

(c) Experiment

Fig. 18: Specimen JM at the end of test.

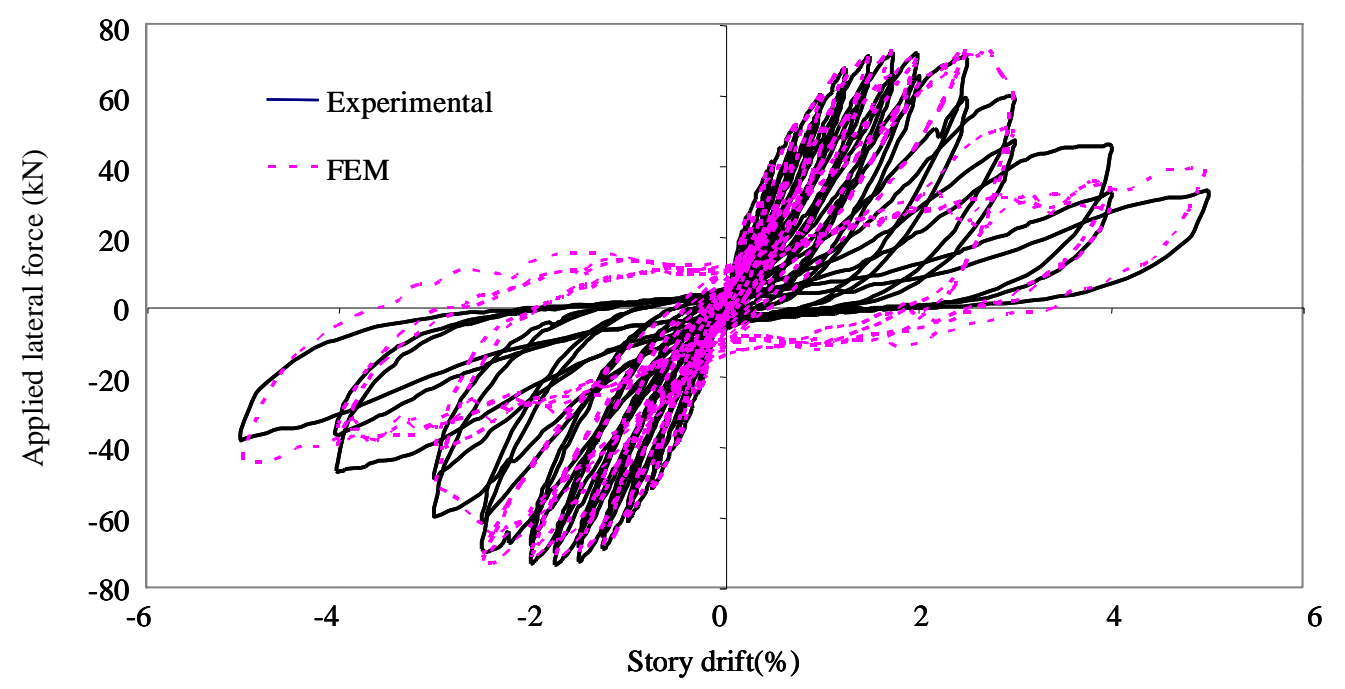

Fig. 19: Relationship between applied load and displacement of Specimen JM.

Before peak, the formation of compressive strut is dominant in all specimens. As the lateral drift increases, the compressive stress rapidly intensifies in specimen JS. The collapse of compressive struts generally coincides with the failure point of the specimens. As for specimen 382 
$\mathrm{JL}$, the compressive strut is maintained throughout the entire loading. At $4.6 \%$ drift when failure took place, the strut remains stable, indicating that the joint is sound and does not cause failure. This observation agrees with the experimental result. This analytical results show that the diagonal strut formation is the principal load resistant mechanism for the joint of specimens JM and JS and the beam flexural yielding is the main load resistant mechanism for specimen JL.

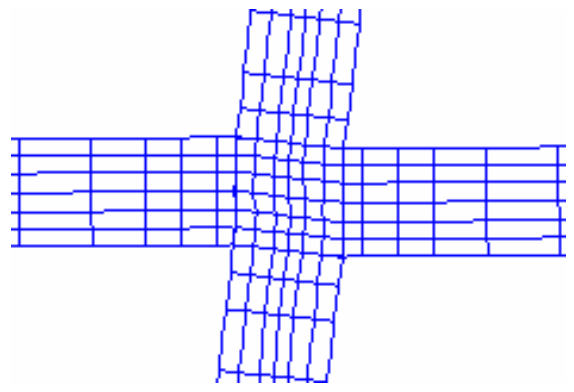

(a) Analytical deformation

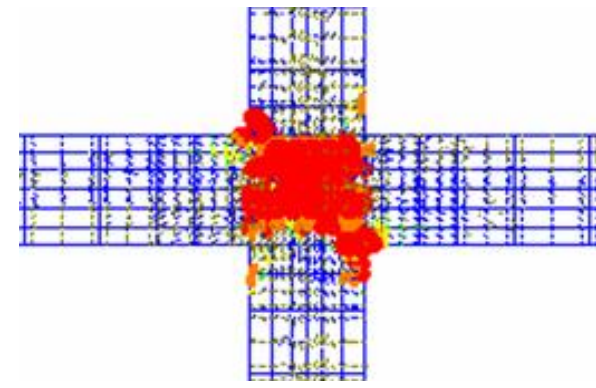

(b) Analytical cracking pattern

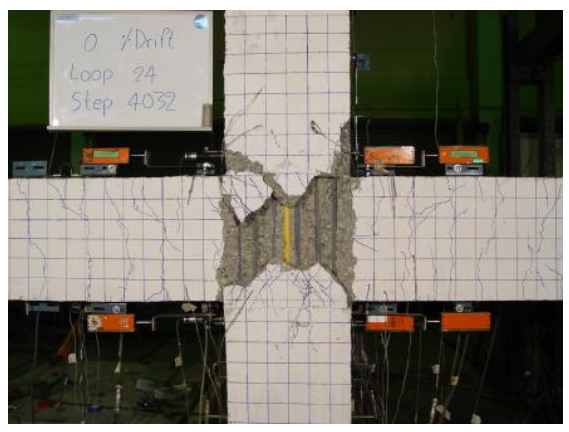

(c) Experiment

Fig. 20: Specimen JS at the end of test.

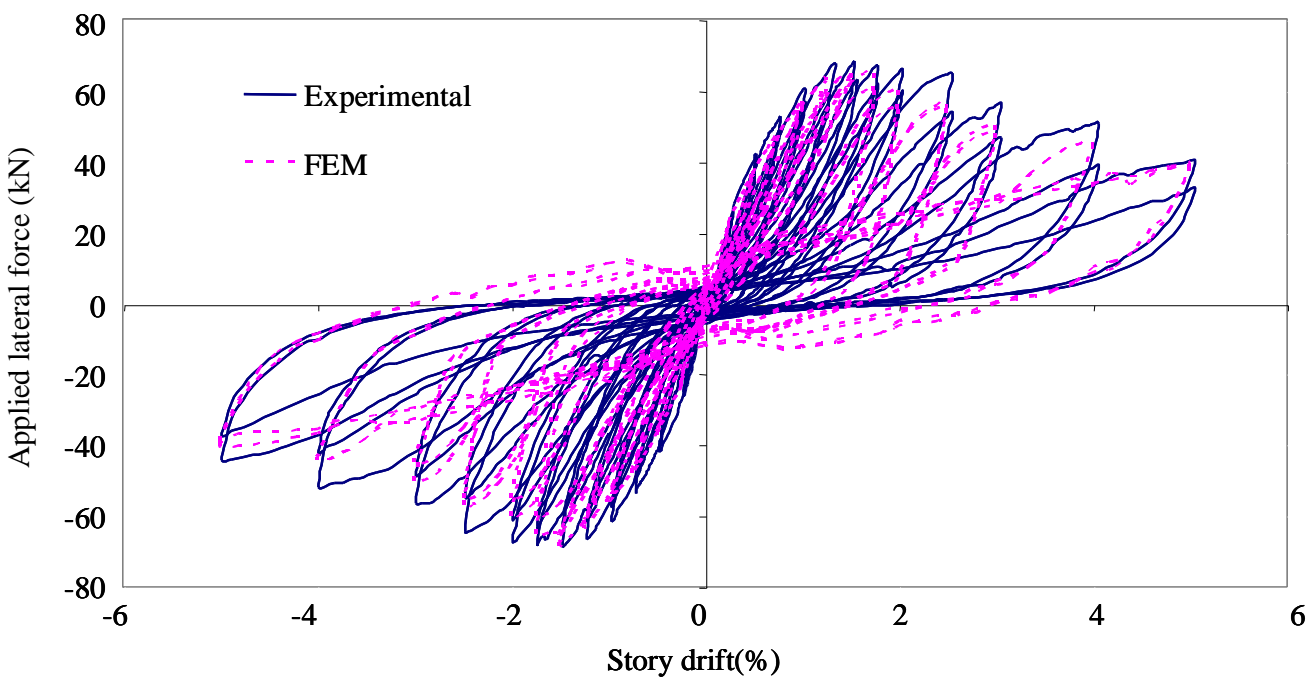

Fig. 21: Relationship between applied load and displacement of Specimen JS. 
Specimen JL

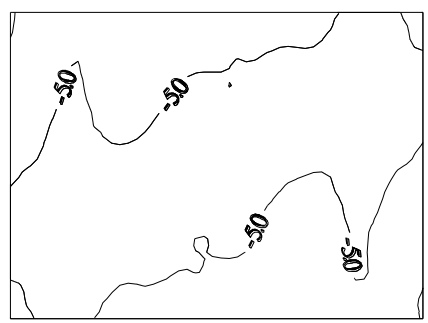

(a) $0.25 \%$ drift

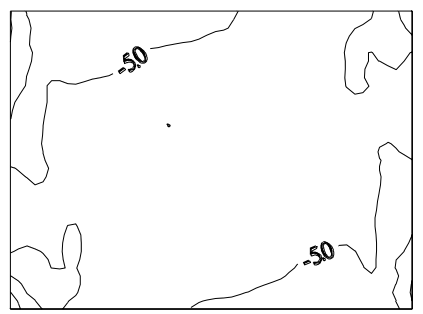

(b) $0.75 \%$ drift

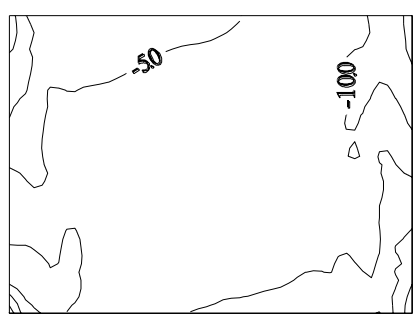

(c) $1.25 \%$ drift

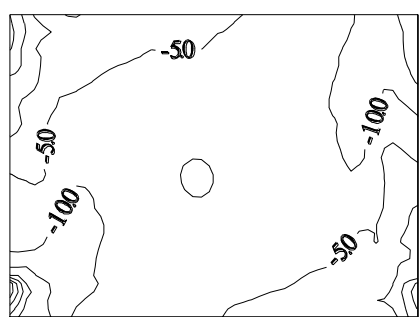

(d) $5.00 \% \mathrm{drift}$
Specimen JM

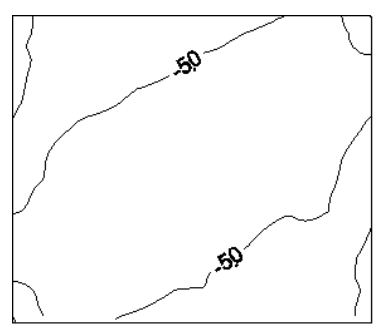

(a) $0.25 \%$ drift

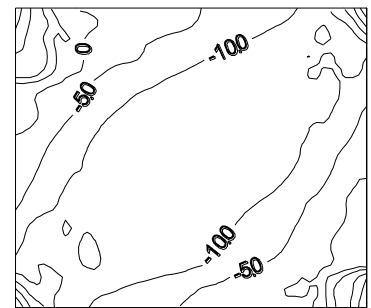

(b) $0.75 \%$ drift

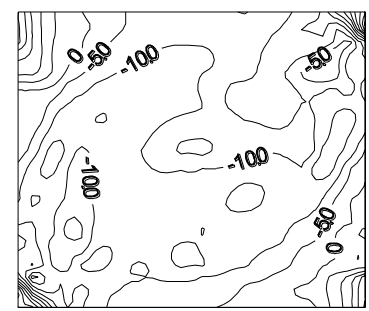

(c) $1.25 \%$ drift

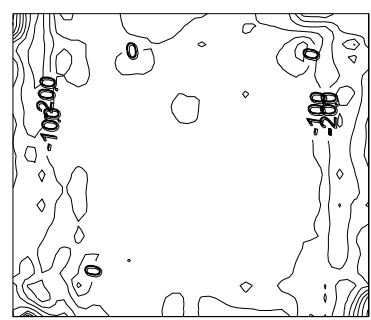

(d) $5.00 \%$ drift
Specimen JS

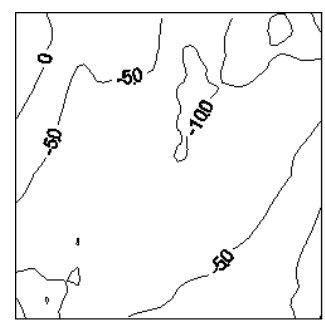

(a) $0.25 \%$ drift

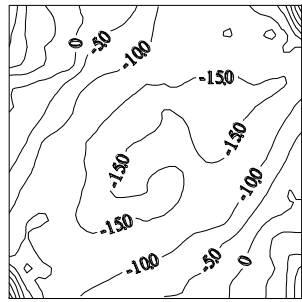

(b) $0.75 \%$ drift

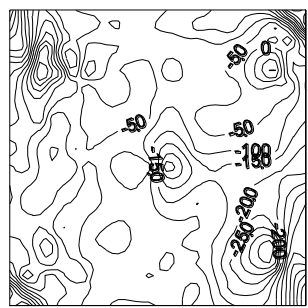

(c) $1.25 \%$ drift

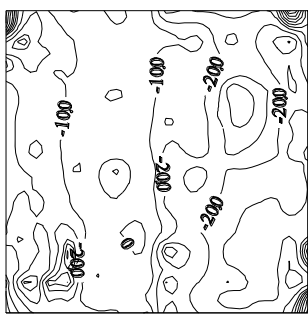

(d) $5.00 \%$ drift

Fig. 22: Principal compressive stresses (unit: MPa) of each specimen.

\section{CONCLUSION}

The paper presents the non-linear finite element analysis as a tool to evaluate the reversed cyclic behavior of sub-standard RC beam-column connections in mid-rise frame building designed and detailed according to the non-seismic provisions of ACI building code in low to moderate seismic region. The finite element analysis includes the nonlinearity of cracked concrete in tension, compression and shear and yielded reinforcement in line with the smeared crack approach. It is shown that the FEM can satisfactorily reproduce the load-displacement envelope as well as hysteretic loops of the tested specimens. As for the failure mode, the finite element 
analysis predicts a ductile beam flexural failure for specimen JL representing actual frame with large column tributary area, and brittle joint shear failure for specimens JM and JS representing actual frame with small and medium column tributary area. The collapse of compressive diagonal strut is verified to be the principal failure mechanism. The analytical failure modes, damage zones and cracking process compare well with the experimental results. However, the analytical hysteretic loops are comparatively wider than experimental ones since the nonlinearity due to concrete spalling and reinforcement buckling has not been taken into account in the present analysis.

Table 1: Comparison between analytical(FEM) and experimental force and displacement (drift) relations.

\begin{tabular}{llll}
\hline Specimen & Force / Drift & FEM analysis & Experiment \\
\hline \multirow{2}{*}{$\mathrm{JL}$} & Maximum force $(\mathrm{kN})$ & 90 & 92 \\
& Drift $(\%)$ & 4.6 & 4.6 \\
$\mathrm{JM}$ & Maximum force $(\mathrm{kN})$ & 73 & 72 \\
& Drift $(\%)$ & 2.0 & 1.75 \\
$\mathrm{JS}$ & Maximum force $(\mathrm{kN})$ & 70 & 68 \\
& Drift $(\%)$ & 1.5 & 1.5 \\
\hline
\end{tabular}

\section{ACKNOWLEDGEMENT}

The authors are very grateful to Thailand Research Fund (TRF) for providing the research fund RMU4880022 to carry out the research.

\section{REFERENCES}

1. Warnitchai, P. (2004), Development of seismic design requirements for buildings in Bangkok against the effects of distant large earthquakes. Proceedings of the 13th World Conference on Earthquake Engineering, Vancouver.

2. Aoyama, H. (1981), Amethod for the evaluation of the seismic capacity of existing RC buildings in Japan. Bulletin of the NZ National Society for Earthquake Engineering, pp. 105-130.

3. Fintel, M. (1991), Shear walls-an answer for seismic resistance?. Construction International, pp. 48-53.

4. Bing, L. and Tso-Chien, P. (2004), Seismic performance of reinforced concrete frames under low intensity earthquake effects. Proceedings of the 13th World Conference on Earthquake Engineering, Vancouver.

5. Warnitchai, P., Pimanmas, A., and Thinth, D.T. (2004), Seismic performance of RC subassemblages with non-seismic reinforcement details. Proceedings of the First Asian Conference for Earthquake Engineering, Phillipines.

6. Maekawa, K., Pimanmas, A., and Okamura, H. (2003), Nonlinear mechanics of reinforced concrete. Son Press.

7. Vecchio, F.J. and Collins, M.P. (1986), The modified compression field theory for reinforced concrete elements subjected to shear. ACI Structural Journal, vol. 83(2), pp. 219231. 
8. Vecchio, F.J. and Collins, M.P. (1988), Predicting the response of reinforced concrete beams subjected to shear using the modified compression field theory. ACI Structural Journal, vol. 85(4), pp. 258-268.

9. Salem, H. and Maekawa, K. (2002), Spatially averaged tensile mechanics for cracked concrete and reinforcement under highly inelastic range, $\mathrm{J}$ Materials Conc Struct Pavements, JSCE, vol. 613(42), pp. 227-293. 